\title{
Analytical Model for Availability Assessment of Large-Scale Offshore Wind Farms including their Collector System
}

\author{
Gayan Abeynayake, Graduate Student Member, IEEE, Tom Van Acker, Dirk Van Hertem, Senior Member, IEEE, \\ and Jun Liang, Senior Member, IEEE
}

\begin{abstract}
With the increase of offshore wind farm size, the use of classical analytical reliability methods becomes computationally intractable. This paper proposes a holistic approach combining multi-state Markov processes and the universal generating function for the availability assessment of radial largescale offshore wind farms. The proposed model combines multistate wind turbine output, wind turbine reliability, and interarray cable reliability models to assess the wind farm output at the point of common coupling. A strategy is developed to split the network into its feeders while still accounting for the wind turbine output dependence, significantly reducing the computational burden. Although the failure rates of inter-array cables are low, their inclusion is pertinent given high repair times and impact on wind farm output given the radial topology of the collection system. A case study on the Anholt wind farm indicates the necessity of accounting for the collection system, showing a significant reduction of $12 \%$ in generation ratio availability for a generation ratio criterion of $95 \%$.
\end{abstract}

Index Terms-Multi-state Markov model, offshore wind, radial systems, availability, reliability, universal generating function.

\begin{tabular}{cl} 
Sets & \multicolumn{1}{c}{ NomENCL } \\
$\mathcal{C}$ & cable set \\
$\mathcal{E}$ & element set \\
$\mathcal{F}$ & failure set \\
$\Phi$ & feeder set \\
$\mathcal{S}$ & state set \\
$\mathcal{S}^{\text {red }}$ & reduced state set \\
$\mathcal{U}$ & user set \\
$\mathcal{W}$ & wind turbine set
\end{tabular}

Variables and Measures

$\begin{array}{ll}X(t) & \text { random variable } \\ \mathbb{P} & \text { probability measure, alternatively denoted as } p \\ t & \text { calendar time } \\ \delta_{s} & \text { initial state probability } \\ \rho_{s \sigma} & \text { transition rate from state } s \text { to } \sigma\end{array}$

This work was supported by the European Commission's Horizon 2020 Research \& Innovation Programme (Marie Skłodowska-Curie Actions) through the project "Innovative Tools for Offshore Wind and DC Grids (InnoDC)", under grant agreement no. 765585.

Gayan Abeynayake and Jun Liang are with the the School of Engineering, Cardiff University, Cardiff, CF24 3AA, United Kingdom. (e-mail: abeynayakepa@cardiff.ac.uk; liangj1@ @ardiff.ac.uk).

Tom Van Acker and Dirk Van Hertem are with the Department of Electrical Engineering, KU Leuven, 3001 Leuven, Belgium. (e-mail: tom.vanacker@esat.kuleuven.be; dirk.vanhertem@esat.kuleuven.be).

(Gayan Abeynayake and Tom Van Acker are co-first authors.)

(Corresponding author: Gayan Abeynayake)

$\begin{array}{ll}\lambda & \text { failure rate } \\ \mu & \text { repair rate } \\ \gamma & \text { ordered tuples of states } \\ v & \text { performance } \\ \omega & \text { universal generating function } \\ \Omega & \text { universal generating operator }\end{array}$

\section{Abbreviations}

WT Wind Turbine

EENS Expected Energy Not Served

GRA Generation Ratio Availability

OWF Offshore Wind Farm

PCC Point of Common Coupling

\section{INTRODUCTION}

\section{A. Background and Motivation}

$\mathbf{P}$ OWER generation from low carbon energy resources has significantly increased in the recent past. Wind power production accounts for a significant share of global renewal production. Wind Europe projects that the installed offshore wind power capacity in Europe will reach $450 \mathrm{GW}$ in 2050 [1], [2]. Compared to its onshore counterpart, an offshore wind farm (OWF) has many advantages, e.g., less visual intrusion, larger generation capacity and non-necessity to reduce turbine noise [3]. However, the expected repair times of OWF components are generally larger, which has a negative impact on its availability [4]. Consequently, while sizing large OWF, its reliability has a greater influence on the final design, making accurate availability assessment even more pertinent.

An OWF is composed of a large number of components, e.g., wind turbines, inter-array cables, busbars, converters, transformers, etc. All these components are comprised of several subsystems, e.g., a typical type-four wind turbine (WT) consists of a generator, drive-train and power converter subsystem. Generally, these subsystems are subject to competing risks, i.e., have different failure characteristics and corresponding repair rates, each with a distinct impact on the components' availability. Therefore, it becomes necessary to assess whether such components should be modeled using a multi-state approach rather than a binary one.

The OWF collector system is made up of a considerable number of inter-array cables. The effectiveness of the OWF to export energy to the point of common coupling (PCC) depends on the reliability of that network. Therefore, it is imperative 
to include the reliability of the collection system in the OWF availability assessment. However, inclusion of the collector system increases the number of components significantly, introducing the dimension curse. Generally, this is addressed by splitting the system into smaller subsystems, assessing them individually before combining them to assess the overall system performance. However, this requires independent components, which no longer holds given the dependency of the wind turbine output, making the inclusion of the collection system in $\mathrm{OWF}$ availability assessment a computationally hard problem. Consequently, the use of a multi-state approach for the availability assessment of OWF combining the inter-array cable network and dependent wind turbine output has not been adequately investigated in the literature.

\section{B. Literature Review}

Accurate availability assessment of large-scale OWF requires including: (a) individual collector system cable reliability, (b) individual wind turbine reliability, and (c) stochastic output of wind turbines dependent on a single stochastic source: the wind, geographically distributed over the network. However, only edge cases have been thoroughly investigated in the literature, including:

1) independent sources, geographically distributed over a network, [5]-[13] i.e., including features (a) and (b), and

2) dependent sources without considering the network, [14][25] i.e., including features (b) and (c).

In the literature, the availability of an $\mathrm{OWF}$ is assessed using either: (a) chronological simulation methods, or (b) analytical methods based on reliability block diagrams (RBDs) and Markov chain models. Among the chronological simulation methods, Monte-Carlo simulation (MCS) with 1000 iterations has been used in [7], [8], considering the cable reliability. A sequential MCS technique has been proposed in [9] for reliability assessment of OWF using a new synthetic windspeed generator. In [10], the same technique has been used for evaluation of reliability of different offshore HVDC grid configurations. This chronological method, under Markovian assumption, has been used in [3], [11] to evaluate the reliability of OWFs considering severe weather conditions. However, with the increase of the number of components, the cardinality of the overall state space becomes too large and the use of such chronological simulation methods to evaluate the reliability of OWF collector systems becomes computationally inefficient. In [12], [14], a RBD and minimal path technique based method has been used to evaluate the reliability of a hypothetical OWF network. Further, the network reduction technique has been considered in [13] to evaluate different OWF topologies. However, this approach does not include the WT and multiple failure events are not considered. Accurate assessment of the OWF availability, e.g., using the generation ratio availability (GRA) index, requires consideration of failure of multiple components. In [5], [6] the offshore sub-sea cable failure has been considered for cable network optimization. The main objective of these studies is to minimize the overall cable investment cost. The problem is solved using a two-stage scenario tree technique and each system state assumed to have at most one unavailable component.

In general, conventional generators are driven by independent inputs. In contrast, WT $\$$ can also be considered to operate in a similar manner but depending on some probabilistic distribution of wind speed [15]. Incorporating variability of wind speed and WT reliability in OWF availability assessment has been adequately addressed in the literature. The importance of the use of multi-state wind speed models was first proposed in [16]. To obtain a finite number of multi-state WT outputs from measured time-series data, different clustering techniques based on minimum Euclidean distance can be used [15]-[17]. In [15], [17]-[19] the wind speed variability is considered together with WT reliability when evaluating the availability of an OWF. However, the inter-array cable network reliability is not considered in any of these studies.

The influence of environmental effects on the availability of OWFs has been evaluated using the common-cause failure method in [20], [21]. The failure causes due to continuous operation of an OWF under higher wind speed conditions have been considered. However, this analysis only considered the first-order component failures and neither network nor the wind speed stochasticity has been taken into account.

In [19], a multi-state system (MSS),WT] model has been developed based on the capacity outage probability table (COPT) to calculate frequency-based reliability indexes. However, network reliability is not included in this COPT approach. Alternatively, in [16], [18] an analytical method based on a binary birth-death Markov process has been used for reliability assessment of wind farms including the multi-state wind farm output. In addition to the Markov model, Auto Regressive Moving Average (ARMA) model is another useful tool which can be used for chronological availability assessment of OWF [22]-[24]. However, heavy computation overhead and dependency on large amount of wind speed data for training ARMA parameters are some of its inherent drawbacks. In all of these works, the WT has been modelled with binary birth-death Markov process and inter-array cable network reliability is not considered. However, when using average failure and repair rates in binary birth-death Markov reliability modelling, information on individual failure and repair rates of component sub-assemblies are required [25].

\section{Contributions and Outline}

This paper aims at filling two research gaps, improving wind farm availability assessment:

1) The impact of (a) dependent wind turbine output, (b) wind turbine reliability, and (c) collection system reliability, on the wind farm availability is considered. The universal generating function (UGF) approach is used to combine the state space of multiple components, overcoming the dimension curse. However, it assumes that the components are independent, which does not hold in this context given the wind turbine output dependence. To this end, a strategy is developed around the UGF approach that allows splitting the network into its feeders while still accounting for the wind turbine output dependence, significantly reducing the computational burden. 
2) Multi-state Markov models are used to represent the reliability of $\mathrm{OWF}$ components, e.g., wind turbines, interarray cables. All these components are comprised of several subsystems, which all have different failure characteristics and corresponding repair rates. Therefore, it becomes pertinent to investigate the error of modeling wind farm components using a binary Markov model rather than a multi-state one, in a Markovian context.

The impact of both aspects is demonstrated on a real-life case study: the Anholt wind farm, which comprises 111 wind turbines and $177 \mathrm{~km}$ inter-array cables.

The paper is organized as follows: Section III presents the mathematical framework. First, the multi-state timehomogeneous Markov process is introduced, which describes the stochastic behavior of the OWF components. Second, the stochastic behavior of the individual OWF components is discussed. Third, a strategy is introduced based on the UGF approach enabling OWF reliability assessment in a tractable way, while accounting for wind turbine output dependence. Section III introduces a case study on a realistic wind farm demonstrating both contributions. Finally, Section IV concludes the paper.

\section{Mathematical Framework}

This section discusses the proposed method, consisting of two parts:

(a) the availability assessment of the individual components using a time-homogeneous multi-state Markov process, described in Section $[\mathrm{II}-\mathrm{A}$ and $\mathrm{II}-\mathrm{B}$, and

(b) the wind farm availability assessment at the point of common coupling using an UGF approach, combining the availability of the individual components, described in Section $\amalg-\mathrm{C}$

Both techniques are well-known in the literature. However, the standard UGF technique assumes independent components, which does not hold for wind turbines given their dependence on a single stochastic source, i.e., the wind speed. This section presents a strategy enhancing the UGF technique enabling dependent sources geographically distributed in a network. Using this strategy, the overall problem can be decoupled into smaller parts, similar to the UGF technique for independent components, significantly reducing the computational burden. In this paper, the proposed strategy is applied to radial largescale offshore wind farm, as this is the most common network structure for their collector systems [26]. However, the method is also effective for other network topologies with dependent sources, e.g., nested networks, as long as the considered network, excluding the sources, is divisible into independent parts.

\section{A. Time-Homogeneous Multi-State Markov Process}

A time-homogeneous Markov process describes a random variable $X(t)$, where $t \geq 0$ denotes the calendar time. The possible values of $X(t)$ are represented by a finite discrete state-space $\mathcal{S}$. A Markov process respects the memorylessness property which entails that a subject's future state solely depends on its current state [27]. We define $\mathscr{F}_{X(\tau)}$ to contain all information with respect to the history of $X$ up to a time $\tau \leq t$ :

$$
\mathbb{P}\left(X(t)=s \mid \mathscr{F}_{X(\tau)}\right)=\mathbb{P}(X(t)=s \mid X(\tau)), \forall s \in \mathcal{S} .
$$

Furthermore, for the process to be time-homogeneous, the following needs to hold:

$$
\mathbb{P}(X(t)=s \mid X(\tau))=\mathbb{P}(X(t-\tau)=s \mid X(0)), \forall s \in \mathcal{S} .
$$

In a continuous time setting, this entails that the transition rates are constant, i.e., the underlying distribution is exponential.

In this paper, the time-homogeneous Markov process is described as an initial value problem, based on the Kolmogorov forward equations:

$$
\begin{array}{ll}
p_{s}^{\prime}(t)=\sum_{\sigma \in \mathcal{S}} \rho_{\sigma s} p_{\sigma}(t)-\rho_{s \sigma} p_{s}(t), & \forall s \in \mathcal{S}, \\
p_{s}(0)=\delta_{s}, & \forall s \in \mathcal{S},
\end{array}
$$

where $p_{s}(t), \delta_{s}$ and $\rho_{s \sigma}$ respectively denote the state probability, initial state probability and transition rate from state $s$ to $\sigma$.

\section{B. Stochastic Behavior of Wind Farm Components}

1) Wind Turbine Output: In order to model the wind turbine output as a time-homogeneous Markov process, two conditions must be met: (a) the sojourn time of any wind speed state needs to follow an exponential distribution; and (b) the continuous wind speed state-space needs to be translated to a discrete one. Condition (a) is met if the non-stationary effects due to seasonal variation can be neglected [28]. This assumption holds for long-term studies and if the wind speed data set is sufficiently large, i.e., longer than one year. Condition (b) is met through clustering of the wind speed data. In this work, the clustering technique proposed in [15] is used. Each data point belongs to a single cluster $s \in \mathcal{S}^{\text {wto }}$ which is selected based on the minimum Euclidean distance to the cluster center. Two special clusters are introduced for a specific wind turbine:

$s_{1}$ groups all wind speed data below its cut-in speed or above its cut-out speed; and

$s_{n}$ groups all wind speed data corresponding with its rated speed (Fig. 1).

It should be noted that the cluster size does not need to be uniform.

2) Wind Turbine Reliability: The wind turbine reliability model is based on its failure and repair characteristics. Commonly, wind turbine reliability is modeled as a binary Markov process, with averaged failure and repair rates: $\bar{\lambda}$ and $\bar{\mu}$ (Fig. 2a] [15], [16], [18], [19], [28].

Several studies have shown that wind turbine failure modes depend on its size, operation and environmental factors [29], [30]. Furthermore, wind turbine reliability is governed by that of its sub-assemblies, i.e., gearbox (b), generator (g) and power converter (pc). Furthermore, each failure is categorised based on the subsequent corrective maintenance, i.e., minor 


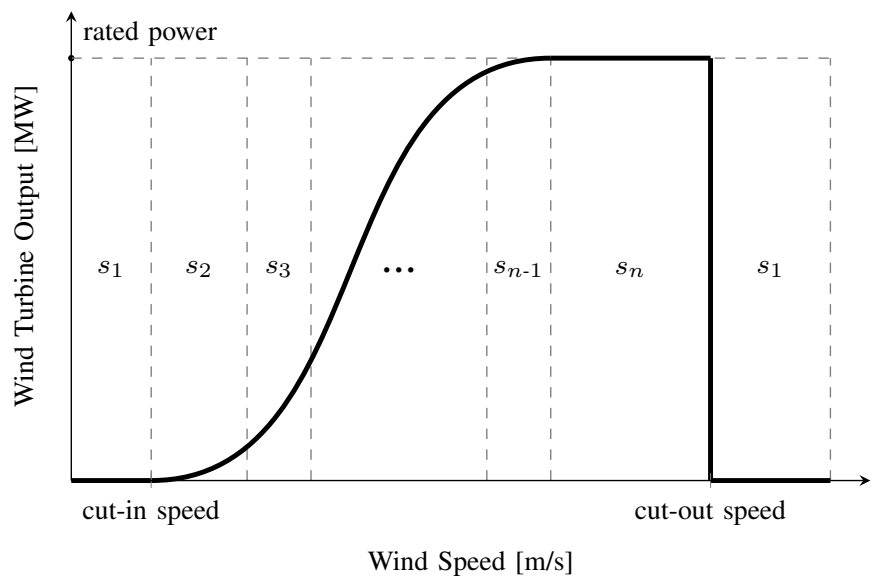

Fig. 1. Illustration of a wind turbine output curve and its discrete statespace $s \in \mathcal{S}^{\text {wto }}$

repair $\left(\mathrm{r}_{1}\right)$, major repair $\left(\mathrm{r}_{2}\right)$ and replacement $\left(\mathrm{r}_{3}\right)$ [31]. Rather than using averaged failure and repair rates, a multi-state system provides a more accurate representation of the failure modes of WT (Fig. 2b] [32]. The failure modes are defined as

$$
\mathcal{F}^{\mathrm{wtr}}=\left\{f^{\mathrm{b}, \mathrm{r}_{1}}, f^{\mathrm{b}, \mathrm{r}_{2}}, f^{\mathrm{b}, \mathrm{r}_{3}}, f^{\mathrm{g}, \mathrm{r}_{1}}, f^{\mathrm{g}, \mathrm{r}_{2}}, f^{\mathrm{g}, \mathrm{r}_{3}}, f^{\mathrm{pc}, \mathrm{r}_{1}}, f^{\mathrm{pc}, \mathrm{r}_{2}}, f^{\mathrm{pc}, \mathrm{r}_{3}}\right\} .
$$

The averaged failure and repair rates of the binary system relate to those of the multi-state system as [25]:

$$
\begin{aligned}
\bar{\lambda} & =\sum_{f \in \mathcal{F}^{\mathrm{wtr}}} \lambda_{f} \\
\bar{\mu} & =\bar{\lambda} / \sum_{f \in \mathcal{F}^{\mathrm{wtr}}} \lambda_{f} \mu_{f}^{-1}
\end{aligned}
$$

3) Cable Section Reliability: Sub-sea power cables and terminations are continuously subject to electrical, thermal, mechanical, and environmental stresses. Both Crow-AMSAA [33] as the Weibull distribution [34], [35] have been used to predict the reliability of cables. The Crow-AMSAA model only considers the accumulated failures per year while the Weibull model considers the failure rate of each component sub-system. Therefore, the Crow-AMSAA model is most suitable for mixed failure mode analysis with less failure information about sub-systems while the Weibull distribution is more useful for detailed failure mode representation [36].

Like other power systems assets, the lifetime of subsea cable also follows the well-known bathtub curve, which consists of a burn-in, useful life and wear-out phase [34]. In this work, it is assumed that the sub-sea cables are in their useful life phase, having constant failure rates [37]-[39].

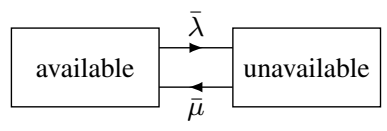

(a) binary system

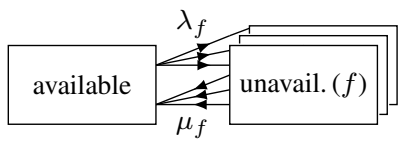

(b) multi-state system
Fig. 2. State-transition diagram of the reliability of (a) binary system, and (b) multi-state system with failures $f \in \mathcal{F}$.
Similar to the wind turbine, a cable system can be divided into sub assemblies: sub-sea cable (c) and its terminations (t). Consequently, its failure modes are defined as

$$
\mathcal{F}^{\mathrm{cbl}}=\left\{f^{\mathrm{c}}, f^{\mathrm{t}}\right\} .
$$

\section{Universal Generating Function Strategy}

In this section, a strategy enhancing the UGF technique is presented to determine the availability of a large-scale OWF The standard UGF technique allows to find the entire multistate system distribution based on the stochastic performance of its elements, i.e., wind turbines, cables, etc., using algebraic procedures [40]. In this section, the UGF technique is extended to account for wind turbine output dependency.

The discrete random variable describing the performance of any element $e \in \mathcal{E}$ can be represented by a UGF

$$
\omega_{e}(z)=\sum_{s \in \mathcal{S}_{e}^{\text {red }}} p_{s} \cdot z^{v_{s}}, \quad \forall e \in \mathcal{E},
$$

where $\mathcal{S}_{e}^{\text {red }}$ represents its reduced state-space with unique performances $v_{s}$ and associated probabilities $p_{s}: \sum_{s \in \mathcal{S}_{\text {red }}} p_{s}=1$. The combined performance of multiple elements with respect to a specific user $u \in \mathcal{U}$ may be found through its universal generating operator (UGO)

$$
\begin{aligned}
\Omega_{u}\left(\left[\omega_{e}(z)\right]_{e \in \mathcal{E}}\right) & =\sum_{s_{1}}^{\mathcal{S}_{e_{1}}^{\text {red }}} \ldots \sum_{s_{|\mathcal{E}|}}^{\mathcal{S}_{|\mathcal{E}|}^{\text {red }}}\left(p_{s_{1}} \cdot \ldots \cdot p_{s_{|\mathcal{E}|}}\right) \cdot z^{f^{\mathrm{str}}\left(v_{s_{1}}, \ldots, s_{|\mathcal{E}|}\right)}(9) \\
& =\sum_{\gamma \in \Gamma} f^{\mathrm{prb}}(\gamma) \cdot z^{f^{\mathrm{str}}(\gamma)} \\
& =\omega_{u}(z)
\end{aligned}
$$

where $\Gamma$ denotes the Cartesian product of the relevant elements' reduced state space: $\Gamma=\prod_{e \in \mathcal{E}} \mathcal{S}_{e}^{\text {red }}$. Its members $\gamma$ are ordered tuples of states $\left(s_{e}\right)_{e \in \mathcal{E}}$, each corresponding to a specific element's reduced state-space $\mathcal{S}_{e}^{\text {red }}$. The probability function $f^{\text {prb }}(\gamma)$ gives the product of state probabilities corresponding to a tuple $\gamma$ :

$$
f^{\mathrm{prb}}(\gamma)=\prod_{s_{e} \in \gamma} p_{s_{e}} .
$$

The structure function $f^{\text {str }}(\gamma)$ expresses the resulting performance towards the user corresponding to a tuple $\gamma$ [40]. Illustrative examples of appropriate structure functions for wind farms are included later in this section.

Given the large number of elements in a realistic OWF it is computationally intractable to solve the entire system directly using the UGF method. To put this into context: a wind farm with 100 binary elements, i.e., $\left|\mathcal{S}_{e}\right|=2$ and $|\mathcal{E}|=100$, amounts to $2^{100} \approx 1.25 \mathrm{e} 30$ possible combinations. Given the radial nature of the collector system, the obvious strategy to reduce the computational burden would be to split it in its feeders $\phi \in \Phi$, evaluate them separately before determining their overall impact on the user's performance. Continuing on the previous example, if the wind farm would consist of ten feeders, the number of combinations would be reduced to $10 \cdot 2^{10}=10240$. However, such an approach is impossible due to the wind turbine output dependency, i.e., it should be the 
same over all feeders which cannot be enforced if the feeders are evaluated separately. The following strategy circumvents the wind turbine output dependency and enables treating it as an independent problem:

1) temporarily replace the wind turbine output UGF by $\omega_{w}(z)=1.0 \cdot z^{1.0}$

2) evaluate the performance of the wind farm collector system as $\omega^{\text {ntw }}$, possibly splitting it into smaller independent parts; and

3) multiply $\omega^{\text {ntw }}$ with the original wind turbine output UGF.

This strategy holds if all other components are binary components, meaning they are either unavailable of available, and, if available, their capacity exceeds that of the wind turbines connected.

To put this into context, consider an illustrative wind farm with four wind turbines and four cables (Fig. 3). The stochastic behavior of a wind turbine output $w$ and cable $c$ are described by:

$$
\begin{array}{ll}
\omega_{w}(z)=0.3 z^{0 \mathrm{MW}}+0.7 z^{2 \mathrm{MW}}, & \forall w \in \mathcal{W}, \\
\omega_{c}(z)=0.1 z^{0 \mathrm{MW}}+0.9 z^{4 \mathrm{MW}}, & \forall c \in \mathcal{C} .
\end{array}
$$

The structure function of the wind farm with respect to the $\mathrm{PCC}$ is given by:

$$
\begin{aligned}
f^{\operatorname{str}}(\gamma)= & \min \left(v_{c_{2}}, v_{w}+\min \left(v_{c_{1}}, v_{w}\right)\right)+ \\
& \min \left(v_{c_{4}}, v_{w}+\min \left(v_{c_{3}}, v_{w}\right)\right) .
\end{aligned}
$$

Given the wind turbine output dependence, all wind turbine's performances may be replaced by a single performance $v_{w}$ at a specific time instance. Consequently, the Cartesian product $\Gamma$ of the components' state-spaces contains $2^{1} \cdot 2^{4}=32$ tuples. The performance of the wind farm at the $\mathrm{PCC}$ is given by:

$$
\begin{aligned}
\omega^{\mathrm{pcc}}(z)= & 0.307 \cdot z^{0 \mathrm{MW}}+0.0126 \cdot z^{2 \mathrm{MW}}+0.11907 \cdot z^{4 \mathrm{MW}}+ \\
& 0.10206 \cdot z^{6 \mathrm{MW}}+0.45927 \cdot z^{8 \mathrm{MW}} .
\end{aligned}
$$

The full calculation is left to the reader.

Alternatively, using the presented strategy, the feeders could be evaluated separately, each using their own structure function:

$$
f_{\phi_{1} / \phi_{2}}^{\mathrm{str}}(\gamma)=\min \left(v_{c_{2} / c_{4}}, v_{w}+\min \left(v_{c_{1} / c_{3}}, v_{w}\right)\right)
$$

Conform the strategy, the UGF of the wind turbine output is temporarily replaced by $\omega_{w}(z)=1.0 z^{1 \mathrm{MW}}$. Consequently,

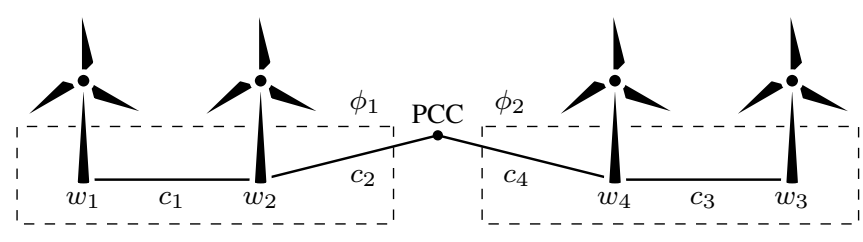

Fig. 3. Wind farm with four wind turbines $w_{1}-w_{4}$ and cables $c_{1}-c_{4}$. given their symmetry, both feeder's $\mathrm{UGF} \omega_{\phi}(z)$ is given by:

$$
\begin{aligned}
\omega_{\phi}(z)= & 0.1 \cdot 0.1 \cdot 1.0 \cdot z^{\min (0 \mathrm{MW}, 1 \mathrm{MW}+\min (0 \mathrm{MW}, 1 \mathrm{MW}))}+ \\
& 0.9 \cdot 0.1 \cdot 1.0 \cdot z^{\min (0 \mathrm{MW}, 1 \mathrm{MW}+\min (4 \mathrm{MW}, 1 \mathrm{MW}))+} \\
& 0.1 \cdot 0.9 \cdot 1.0 \cdot z^{\min (4 \mathrm{MW}, 1 \mathrm{MW}+\min (0 \mathrm{MW}, 1 \mathrm{MW}))}+ \\
& 0.9 \cdot 0.9 \cdot 1.0 \cdot z^{\min (4 \mathrm{MW}, 1 \mathrm{MW}+\min (4 \mathrm{MW}, 1 \mathrm{MW}))}(18) \\
= & 0.1 \cdot z^{0 \mathrm{MW}}+0.09 \cdot z^{1 \mathrm{MW}}+0.81 \cdot z^{2 \mathrm{MW}} .
\end{aligned}
$$

Combining both feeders $\phi_{1}$ and $\phi_{2}$ results in:

$$
\begin{aligned}
\omega^{\mathrm{ntw}}(z)= & 0.01 \cdot z^{0 \mathrm{MW}}+0.018 \cdot z^{1 \mathrm{MW}}+0.1701 \cdot z^{2 \mathrm{MW}}+ \\
& 0.1458 \cdot z^{3 \mathrm{MW}}+0.6561 \cdot z^{4 \mathrm{MW}} .
\end{aligned}
$$

After combination with the original wind turbine output UGF $\omega_{w}(z)$, the performance of the wind farm at the PCC becomes:

$$
\omega^{\mathrm{pcc}}(z)=0.307 \cdot z^{0 \mathrm{MW}}+0.0126 \cdot z^{2 \mathrm{MW}}+0.11907 \cdot z^{4 \mathrm{MW}}+
$$$$
0.10206 \cdot z^{6 \mathrm{MW}}+0.45927 \cdot z^{8 \mathrm{MW}} \text {. }
$$

Comparing (16) with 21, it can be seen that the proposed strategy gives the same solution, whereas it only required the evaluation of $2 \cdot 2^{2}+3^{2}+2 \cdot 5=27$ combinations, relative to the 32 combinations of the brute-force approach. Although the difference seems small in this particular case, it circumvents the exponential growth in combinations when the wind farm size becomes realistic. To illustrate this exponential growth, consider a network of variable size consisting of binary components (Fig. 4). Depending on the number of independent subsystems $n$, the number of possible combinations varies. Two trends can be seen:

(a) increasing the subsystems for large scale problems reduces the number of combinations exponentially; and

(b) increasing the subsystems for small scale problems increases the number of combinations.

\section{Reliability Indices}

Two indices, i.e., generation ratio availability (GRA) and expected energy not served (EENS), are introduced to show the impact of the contributions. GRA is defined as the probability that at least a given percentage of the wind power can be transferred to the PCC [41]. That percentage is referred to as the generation ratio criterion (GRc). For example, for a

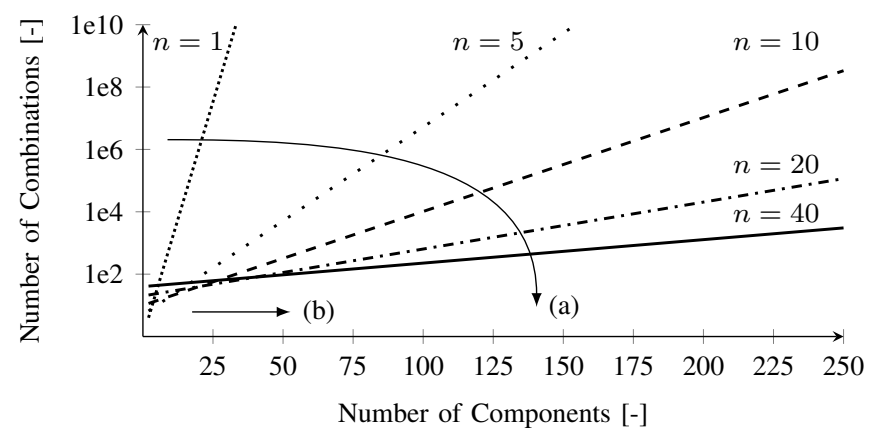

Fig. 4. Illustration of the scalability of the UGF technique on a network of variable size consisting of binary components, considering different number of independent subsystems $n$. 
GRc of $70 \%$ in given wind conditions which result in total wind power output of $200 \mathrm{MW}$, the corresponding GRA gives the percentage that at least $140.0 \mathrm{MW}$ can be transferred to the PCC. As a result, the GRA is not subject to wind speed variability and therefore is a very appropriate index to show the impact of network reliability. The GRA for a given GRc can be determined using $\omega^{\text {ntw }}$ as

$$
\begin{aligned}
\mathrm{GRA}(\mathrm{GRc}) & =\sum_{i \in \mathcal{I}: \mathrm{cnd}_{i}} \omega^{\mathrm{ntw}} \cdot p_{i}, \\
\operatorname{cnd}_{i} & =\omega^{\mathrm{ntw}} \cdot v_{i} \geq \mathrm{GRc} \cdot \max \left(\omega^{\mathrm{ntw}} \cdot v\right),
\end{aligned}
$$

where $\mathcal{I}$ gives the set of all elements of UGF $\omega^{\text {ntw }}$. The condition $\mathrm{cnd}_{i}$ evaluates whether the corresponding value of the UGF $\omega^{\text {ntw }} \cdot v_{i}$ is greater or equal to $\mathrm{GRc}$ To put this into context, following the illustration given in Section II-C, a GRc of 0.7 and network UGF.

$$
\begin{aligned}
\omega^{\mathrm{ntw}}(z)= & 0.01 \cdot z^{0 \mathrm{MW}}+0.018 \cdot z^{1 \mathrm{MW}}+0.1701 \cdot z^{2 \mathrm{MW}}+ \\
& 0.1458 \cdot z^{3 \mathrm{MW}}+0.6561 \cdot z^{4 \mathrm{MW}} .
\end{aligned}
$$

results in a condition $\mathrm{cnd}_{i}$ for a specific performance $v_{i}$ :

$$
\operatorname{cnd}_{i}=\omega^{\mathrm{ntw}} \cdot v_{i} \geq 0.7 \cdot \max \left(\omega^{\mathrm{ntw}} \cdot v\right)=0.7 \cdot 4.0=2.8,
$$

and consequently gives a generation ratio availability:

$$
\operatorname{GRA}(0.7)=0.1458+0.6561=0.8019 \text {. }
$$

The EENS index gives the expected yearly energy that cannot be delivered to the PCC with respect to the maximum yearly energy of the wind farm. In contrast to GRA, EENS incorporates the wind speed variability. The EENS can be determined using $\omega^{\mathrm{pcc}}$ as:

$$
\text { EENS }=8760 \cdot \sum_{i \in \mathcal{I}} \omega^{\mathrm{pcc}} \cdot p_{i} \cdot\left(\max \left(\omega^{\mathrm{pcc}} \cdot v\right)-\omega^{\mathrm{pcc}} \cdot v_{i}\right)(25)
$$

To put this into context, following the illustration given in Section III-C, a point of common coupling UGF

$$
\begin{aligned}
\omega^{\mathrm{pcc}}(z)= & 0.307 \cdot z^{0 \mathrm{MW}}+0.0126 \cdot z^{2 \mathrm{MW}}+0.11907 \cdot z^{4 \mathrm{MW}}+ \\
& 0.10206 \cdot z^{6 \mathrm{MW}}+0.45927 \cdot z^{8 \mathrm{MW}},
\end{aligned}
$$

results in expected energy not served:

$$
\begin{aligned}
\text { EENS }=8760 \cdot( & 0.307 \cdot(8-0)+0.0126 \cdot(8-2)+ \\
& 0.11907 \cdot(8-4)+0.10206 \cdot(8-6))+ \\
& 0.45927 \cdot(8-8)) \\
= & 8760 \cdot(2.456+0.0756+0.47628+0.20412+0.0) \\
= & 28137.12 \mathrm{MWh} .
\end{aligned}
$$

\section{CASE STUdY}

In this section, a case study is presented for the Anholt offshore wind farm located between Djursland and Anholt island in Denmark [42]. The Anholt wind farm comprises 111 wind turbines and a collector system consisting of $177 \mathrm{~km}$ interarray cables (Fig. 5). First, the impact of the collector system reliability is studied. Second, the impact of modeling each component as a multi-state system is discussed. All analyses in this case study are performed using MultiStateSystems.j! a package developed in JULIA to solve multi-state systems [43].

\footnotetext{
${ }^{1}$ Available at https://github.com/timmyfaraday/MultiStateSystems.j1
}

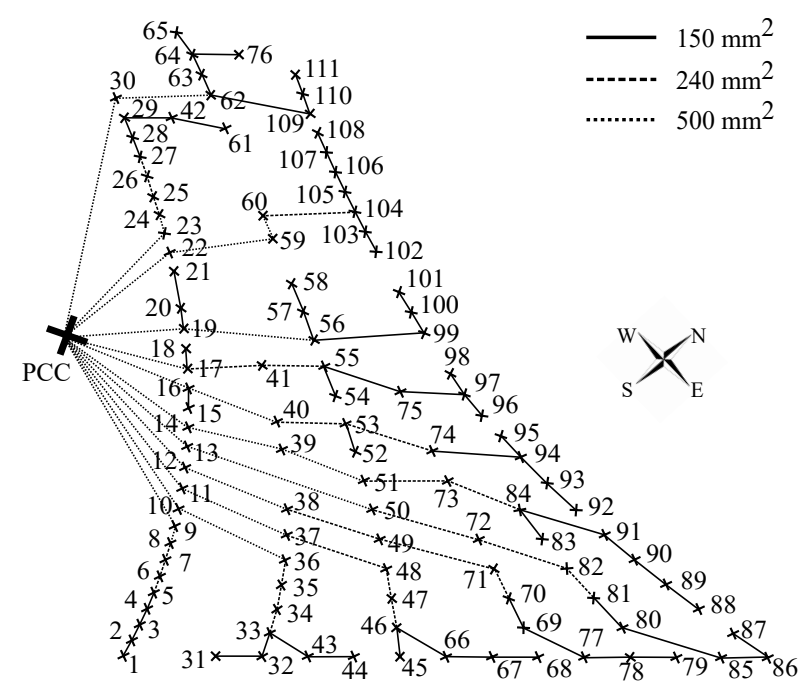

Fig. 5. Inter array cable configuration of the Anholt wind farm.

\section{A. Anholt Wind Farm}

The Anholt wind farm includes 111 Siemens SWT-3.6-120 wind turbines with a rated power of $3.6 \mathrm{MW}$ and a rotor diameter of $120 \mathrm{~m}$, amounting to a nameplate capacity of 399.6 MW. All wind turbines have a cut-in speed of $3.5 \mathrm{~m} / \mathrm{s}$, rated speed of $14.0 \mathrm{~m} / \mathrm{s}$, and cut-off speed of $25.0 \mathrm{~m} / \mathrm{s}$. To determine the wind turbine power output, ten minutes averaged wind speed data from the Anholt wind farm is combined with its power curve [44]. This data set comprises one year of wind speed data, i.e., 52560 wind speed data points. Once the time series of the wind turbine power output is obtained, it is clustered using the method discussed in Section II-B1 An example of seven clusters is shown in Table I including the cluster centers and corresponding transition rate matrix.

As discussed in Section III-B2, wind turbine reliability is governed by that of its sub-assemblies. For the purpose of this case study, the reliability data presented in [31], [32] is used. This data complies with the Anholt wind turbines as it is valid for wind turbines with an output range of 2.0 to 4.0 MW and a rotor diameter range of 80 to $120 \mathrm{~m}$. Table II summarizes the relevant data for each of these sub-assemblies and the corrective maintenance strategies following a specific failure.

The collector system consists of $177 \mathrm{~km}$ of inter-array cables. Each cable segment may have a different length and diameter, depending on the number of connected wind turbines. The Anholt wind farm comprises three different cable diameters, i.e., 150,240 and $500 \mathrm{~mm}^{2}$, with corresponding failure rates [38], [45], [46]. Table [II] summarizes the relevant data.

\section{B. Impact of Collector System Reliability}

The collector system of the Anholt wind farm consists of twelve radial feeders, nine feeders with nine wind turbines and three feeders with ten wind turbines. The lay-out of each feeder is different, resulting in a unique structure function for each feeder. The impact of the collector system is determined using the strategy presented in Section $[\mathrm{II}-\mathrm{C}$ Three scenarios are considered: 
TABLE I

ILLUSTRATION OF Wind TURBINE OUTPUT CLUSTERING FOR SEVEN CLUSTERS INCLUDING THEIR CENTER AND TRANSITION RATES

\begin{tabular}{rrrrrrrrr} 
& & \multicolumn{8}{c}{ Transition Rates $[1 / \mathrm{yr}]$} \\
\cline { 3 - 9 } Cl. & Center & \multicolumn{1}{c}{ [MW] } & \multicolumn{1}{c}{1} & \multicolumn{1}{c}{3} & \multicolumn{1}{c}{4} & \multicolumn{1}{c}{6} & \multicolumn{1}{c}{7} \\
\hline 1 & 0.000 & 0.0 & 5284.8 & 0.0 & 0.0 & 10.7 & 0.0 & 32.2 \\
2 & 0.255 & 2027.6 & 0.0 & 3169.1 & 37.1 & 8.2 & 0.0 & 0.0 \\
3 & 0.858 & 0.0 & 4732.8 & 0.0 & 5105.8 & 146.8 & 12.2 & 0.0 \\
4 & 1.638 & 0.0 & 44.5 & 6325.6 & 0.0 & 6043.8 & 111.2 & 44.5 \\
5 & 2.432 & 9.3 & 0.0 & 74.7 & 7799.4 & 0.0 & 5865.9 & 850.0 \\
6 & 3.198 & 0.0 & 0.0 & 62.4 & 249.6 & 13355.1 & 0.0 & 12190.2 \\
7 & 3.600 & 14.2 & 0.0 & 9.5 & 23.7 & 374.9 & 2838.0 & 0.0 \\
\hline
\end{tabular}

TABLE II

RELIAbiLITy of Wind TURBine Sub-Assemblies

\begin{tabular}{cccc} 
Sub-Assembly & Corr. Maintenance & $\lambda_{f}[1 / \mathrm{yr}]$ & $\mu_{f}[1 / \mathrm{hr}]$ \\
\hline \multirow{3}{*}{ Gearbox (b) } & Minor $\left(\mathrm{r}_{1}\right)$ & 0.059 & 0.0132 \\
& Major ( $\left.\mathrm{r}_{2}\right)$ & 0.042 & 0.0361 \\
& Repl. $\left(\mathrm{r}_{3}\right)$ & 0.432 & 0.0752 \\
\hline \multirow{3}{*}{ Generator (g) } & Minor ( $\left.\mathrm{r}_{1}\right)$ & 0.007 & 0.1695 \\
& Major ( $\left.\mathrm{r}_{2}\right)$ & 0.024 & 0.3704 \\
& Repl. ( $\left.\mathrm{r}_{3}\right)$ & 0.437 & 0.0625 \\
\hline \multirow{3}{*}{ Converter (c) } & Minor ( $\left.\mathrm{r}_{1}\right)$ & 0.077 & 0.0158 \\
& Major ( $\left.\mathrm{r}_{2}\right)$ & 0.338 & 0.0443 \\
& Repl. ( $\left.\mathrm{r}_{3}\right)$ & 0.538 & 0.0515 \\
\hline
\end{tabular}

TABLE III

Reliability of the CABle System

\begin{tabular}{lcccc}
\multirow{2}{*}{ Diameter } & \multicolumn{2}{c}{ Cable } & \multicolumn{2}{c}{ Cable Termination } \\
\cline { 2 - 5 } & $\lambda[1 / \mathrm{yr} / \mathrm{km}]$ & $\mu[1 / \mathrm{hr}]$ & $\lambda[1 / \mathrm{yr}]$ & $\mu[1 / \mathrm{hr}]$ \\
\hline $150 / 240 \mathrm{~mm}^{2}$ & $7.43 \mathrm{e}-3$ & $6.94 \mathrm{e}-4$ & $1.68 \mathrm{e}-3$ & $9.26 \mathrm{e}-4$ \\
$500 \mathrm{~mm}^{2}$ & $9.45 \mathrm{e}-3$ & $6.94 \mathrm{e}-4$ & $1.68 \mathrm{e}-3$ & $9.26 \mathrm{e}-4$ \\
\hline
\end{tabular}

1) fully reliable, both the wind turbine and cable system reliability are neglected;

2) incl. wind turbine reliability, the wind turbine reliability is considered, the cable system is still neglected; and

3) incl. wind turbine and cable system reliability, both the wind turbine and cable system reliability are considered.

All components are modeled as multi-state systems. Using the strategy presented in Section II-C, the state-space $\Gamma$ contains $8,6.14 \mathrm{e} 4$ and $4.40 \mathrm{e} 7$ elements, respectively. A brute-force approach for the third scenario would result in a state-space $\Gamma$ containing 5.39e67 elements. Therefore, applying the proposed strategy reduces the state-space $\Gamma$ with a factor of $1.22 \mathrm{e} 60$. A $3.10 \mathrm{GHz}$ Dual-Core MacBook Pro with $16 \mathrm{~Gb}$ of memory solved the respective scenarios in $0.98 \mathrm{~s}, 10.61 \mathrm{~s}$ and $150.22 \mathrm{~s}$.

Fig. 6 shows the EENS for the three scenarios with respect to the number of wind speed clusters. Two conclusions can be drawn from this figure. First, at least eight clusters are necessary to accurately represent the Anholt wind speed data. Second, not including wind turbine and cable system reliability introduces an error with respect to the EENS: $3.89 \mathrm{GWh} / \mathrm{yr}(0.2 \%)$ and $19.89 \mathrm{GWh} / \mathrm{yr}(1.0 \%)$, respectively. To put this into context, using the average Danish electricity price of $36.57 \$ / \mathrm{MWh}$ (2017) [47], this amounts to $4.70 \mathrm{M} \$$ and $24.05 \mathrm{M} \$$, respectively, over the lifetime of the Anholt wind farm $(20 \mathrm{yr})$ with a discount rate of $5 \%$ [48]. The impact

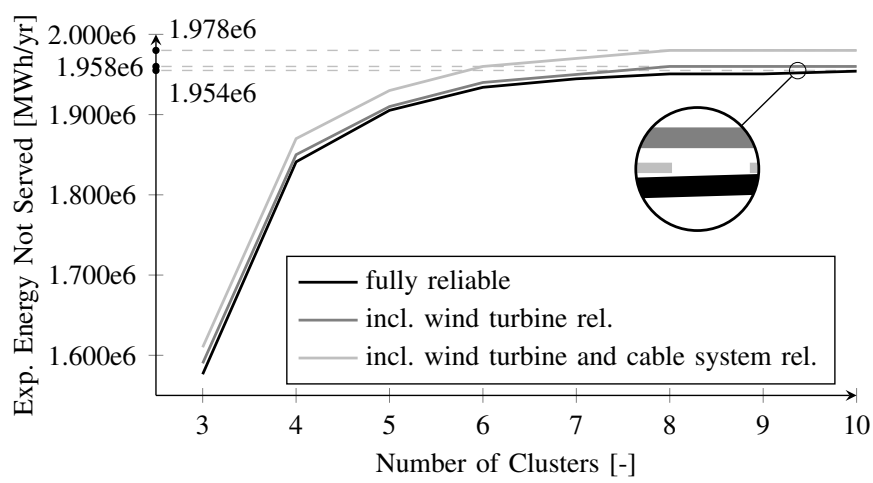

Fig. 6. Expected energy not served for the Anholt wind farm in function of the number of wind speed clusters.

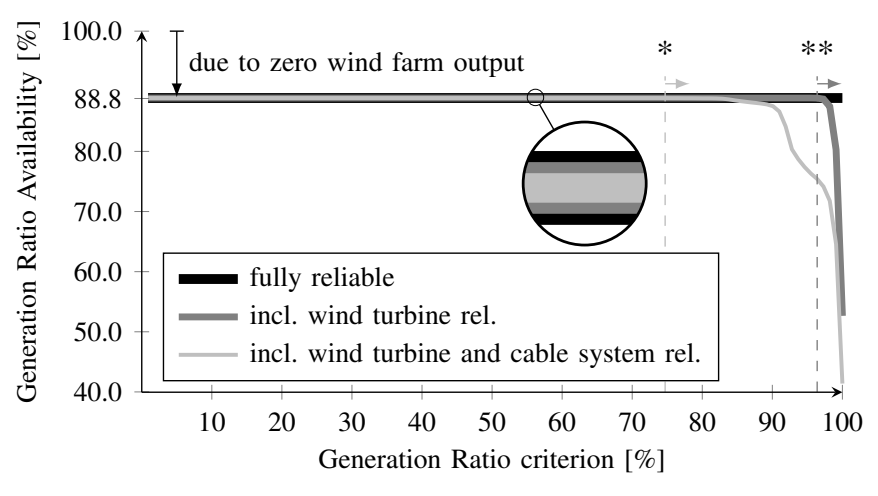

Fig. 7. Generation ratio availability for the Anholt wind farm. The GRc at which the impact of the collector system and wind turbine reliability starts is indicated using * and $* *$, respectively.

of the collector system reliability significantly exceeds that of the wind turbine reliability, i.e, by a factor five.

Fig. 7 shows the GRA for the three scenarios with respect to the GRc. This figure affirms the previous conclusions. For a GRc of $95.0 \%$, the GRA is reduced to $76.3 \%(-12.0 \%)$ when considering the collector system reliability. Furthermore, the figure shows that the impact of collector system reliability starts from a significantly lower GRc compared to the wind turbine reliability: $74.7 \%$ and $96.4 \%$, respectively.

Fig. 8 presents the probability distribution of the power delivered at the PCC for the latter two scenarios: Fig. 8a and Fig. $8 \mathrm{~b}$, respectively. Each dot represents the probability of the wind farm delivering a specific power at the PCC It can be seen that including the cable system reliability in addition to the wind turbine reliability has two effects: 1) the average output for a specific cluster is reduced; and 2) the output variance within a specific cluster is increased. Figure 9 shows the difference between both probability distributions and corroborates this.

\section{Multi-State vs. Binary System Reliability}

As discussed in Section II-B2 multi-state systems can be approximated as binary systems with their averaged failure and repair rates (5)-(6). It should be noted that the failure and repair rates of each sub-assembly are required to determine these averaged rates. To show the difference between multi- 


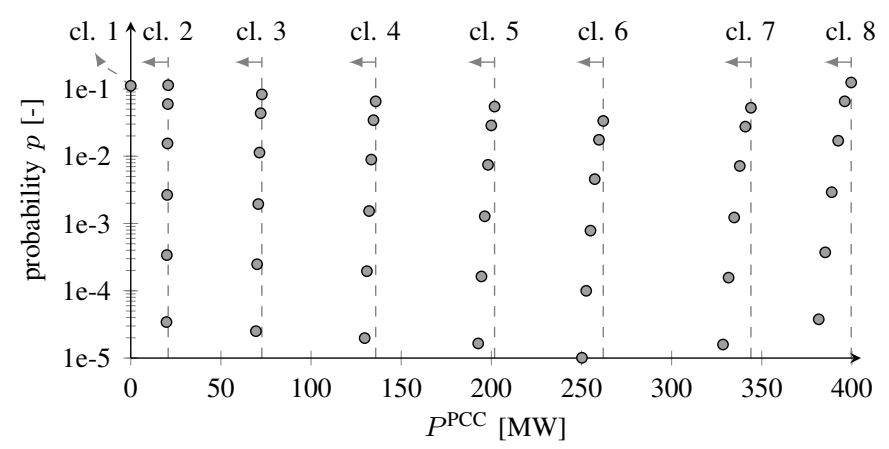

(a) including wind turbine reliability

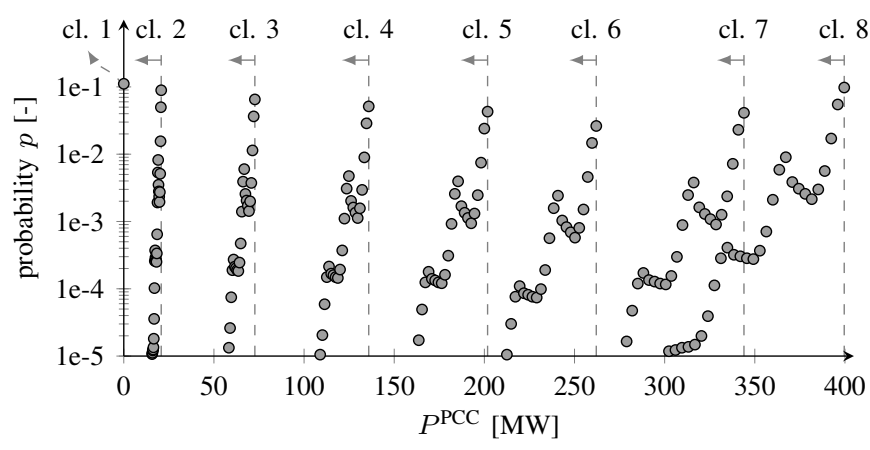

(b) including wind turbine and cable system reliability

Fig. 8. Probability distribution of the power delivered at the PCC for the scenario incl. wind turbine reliability (Fig. $8 \mathrm{a}$ and the scenario incl. wind turbine and cable system reliability (Fig. $8 \mathrm{bb}$. Each dot represents the probability of the wind farm delivering a specific power at the PCC All probabilities $p \leq 1 e-5$ are omitted.

state and binary system modeling for wind farm components in a Markovian context, the latter two scenarios introduced in Section III-B are considered with eight wind speed clusters.

The difference of EENS between multi-state and binary system modeling is about $1.8 \mathrm{MWh} / \mathrm{yr}(0.0001 \%)$ for the scenario including wind turbine and cable system reliability. The EENS difference for the scenario which neglects the inter-array cable network is $3.2 \mathrm{MWh} / \mathrm{yr}(0.0002 \%)$. The GRA values for GRc of $95 \%$ remain unchanged for both scenarios: $88.88 \%$ and $76.32 \%$, respectively.

Fig. 10 illustrates the difference of the probability distributions between multi-state system and binary system modeling for the scenario including wind turbine and cable system reliability. Although there is a variation between the two modeling approaches, the difference is not significant. This is supported by the reliability indices enumerated in the previous paragraph. Accordingly, this analysis suggests that a binary reliability model suffices in a Markovian context to model multi-state $\mathrm{OWF}$ components.

\section{CONCLUSiON}

This paper highlights the importance of the inclusion of inter-array cable network reliability in the availability assess- ment of large-scale offshore wind farms. To evaluate their reliability, an analytical method based on universal generating function combined with multi-state Markov processes is proposed considering its dependencies. This method combines multi-state wind turbine outputs, reliability of different subassemblies of wind turbine and cable systems, each with different failure and repair rate characteristics.

The case study carried out for $400 \mathrm{MW}$ Anholt offshore wind farm in Denmark highlights the requirement for the inclusion of inter-array cable network in the reliability evaluation of such electrical networks. Not including the cable network reliability results in a $12 \%$ error in generation ratio availability for generation ratio criterion of $95 \%$. Furthermore, this could lead to an underestimation in energy not supplied over the operating lifetime of the offshore wind farm worth $24.05 \mathrm{M} \$$. It has been shown that including the cable system reliability is five times more impactful compared to including the wind turbine reliability. In the Markovian context, it has been shown that it is appropriate to represent individual wind farm components using their averaged failure and repair rates.

Finally, in line with the outcome of this study following general suggestions can be made: (a) it is imperative to include the cable network reliability in the availability assessment of

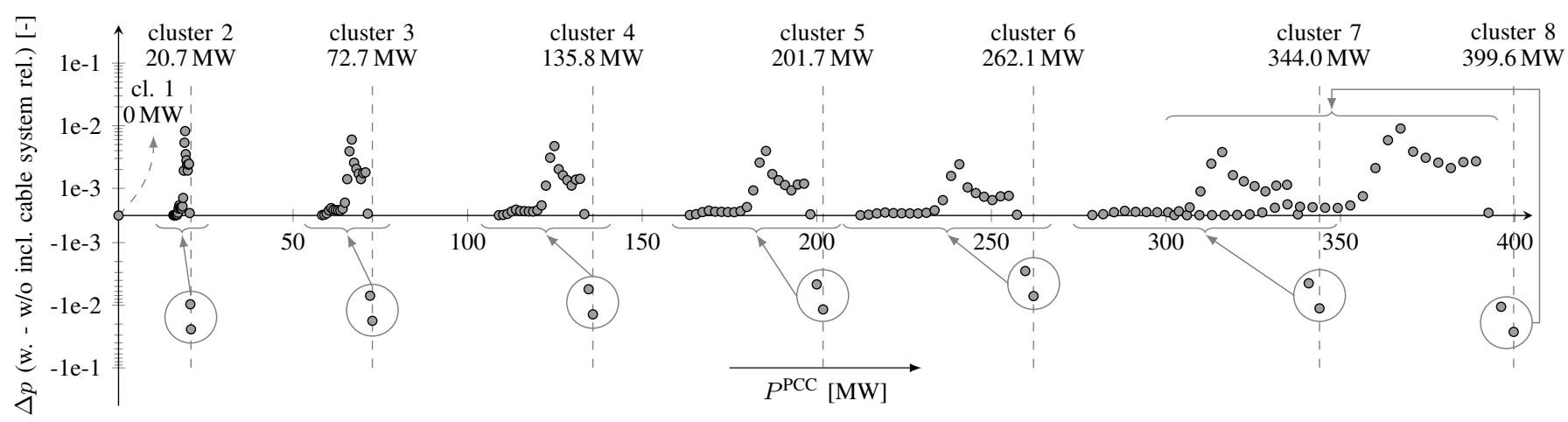

Fig. 9. Difference of the probability distributions between the scenario incl. wind turbine and cable system reliability and the scenario incl. wind turbine reliability. Each dot represents the difference in probability between both scenarios of the wind farm delivering a specific power at the PCC All probability differences $|\Delta p| \leq 1 e-5$ are omitted. 


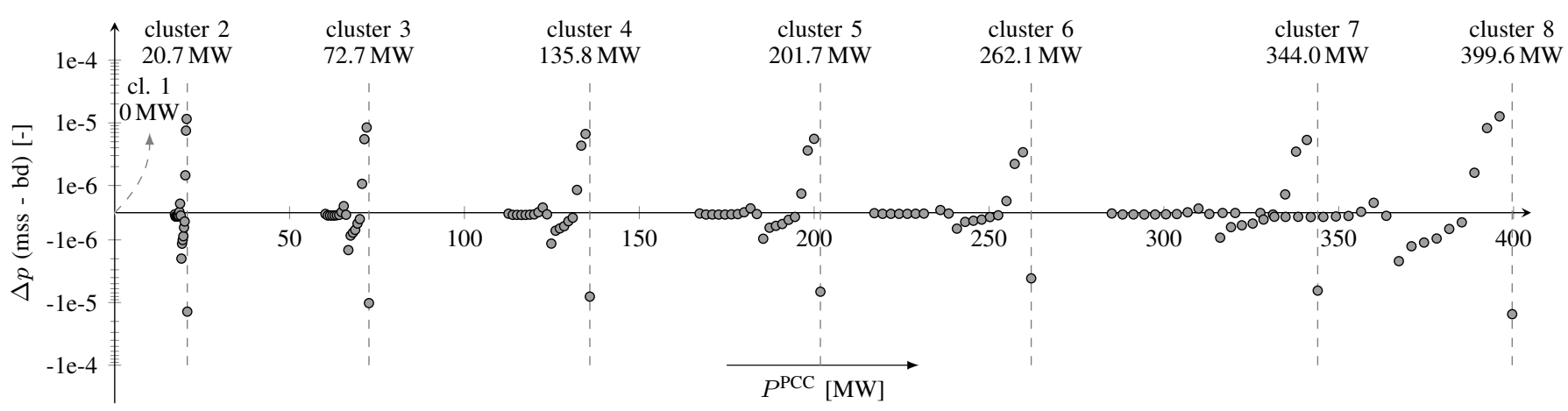

Fig. 10. Difference of the probability distributions between modeling the component reliability as a multi-state system and binary system for the scenario incl. wind turbine and cable system reliability. Each dot represents the difference in probability between reliability models of the wind farm delivering a specific power at the PCC All probability differences $|\Delta p| \leq 1 e-8$ are omitted.

large scale offshore wind farms and the network dependency can be integrated using the proposed analytical method; and (b) if the components hold Markov properties, it is appropriate to represent individual wind farm components using their averaged failure and repair rates with the knowledge of failure and repair rates of associated sub-systems. While this paper only considers Markovian processes, the proposed UGF approach could easily be extended for a non-Markovian context. This is left for future work.

\section{ACKNOWLEDGMENT}

The authors would like to acknowledge the following people for their valuable contributions to the paper: Dr. Juan-Andrés Pérez-Rúa (DTU, Denmark) and Dr. Hakan Ergun (KU Leuven, Belgium)

\section{REFERENCES}

[1] D. Hirst, P. Bolton, and S. Priestley, "Net zero in the UK," Tech. Rep., 2019, Accessed: Jun. 12, 2020. [Online]. Available: https://commonslibrary.parliament.uk/research-briefings/cbp-8590

[2] K. Freeman et al., "Our Energy, Our Future - How offshore wind will help Europe go carbon-neutral," Tech. Rep., 2019, Accessed: Jun. 12, 2020. [Online]. Available: https://windeurope.org/wp-content/uploads/ files/about-wind/reports/WindEurope-Our-Energy-Our-Future.pdf

[3] H. Chao, B. Hu, K. Xie, H. Tai, J. Yan, and Y. Li, "A sequential mcmc model for reliability evaluation of offshore wind farms considering severe weather conditions," IEEE Access, vol. 7, pp. 132 552-132 562, 2019.

[4] C. Dao, B. Kazemtabrizi, and C. Crabtree, "Wind turbine reliability data review and impacts on levelised cost of energy," Wind Energy, 2019.

[5] M. Banzo and A. Ramos, "Stochastic optimization model for electric power system planning of offshore wind farms," IEEE Transactions on Power Systems, vol. 26, no. 3, pp. 1338-1348, 2011.

[6] S. Lumbreras and A. Ramos, "Optimal design of the electrical layout of an offshore wind farm applying decomposition strategies," IEEE Transactions on Power Systems, vol. 28, no. 2, pp. 1434-1441, 2013.

[7] Y. Wu, P. Su, Y. Su, T. Wu, and W. Tan, "Economics- and reliabilitybased design for an offshore wind farm," IEEE Transactions on Industry Applications, vol. 53, no. 6, pp. 5139-5149, 2017.

[8] Y. Wu et al., "Economics- and reliability-based design for an offshore wind farm," in 2017 IEEE/IAS 53rd Industrial and Commercial Power Systems Technical Conference (I\&CPS), 2017, pp. 1-9.

[9] N. Barberis Negra, O. Holmstrom, B. Bak-Jensen, and P. Sorensen, "Aspects of relevance in offshore wind farm reliability assessment," IEEE Transactions on Energy Conversion, vol. 22, no. 1, pp. 159-166, 2007.
[10] C. MacIver, K. R. W. Bell, and D. P. Nedić, "A reliability evaluation of offshore hvdc grid configuration options," IEEE Transactions on Power Delivery, vol. 31, no. 2, pp. 810-819, 2016.

[11] G. Ault and D. Mcmillan, "Specification of reliability benchmarks for offshore wind farms," in Proceedings of ESREL 2008, CRC Press, Valencia, Spain, 2008, pp. 2601-2606.

[12] O. Dahmani, S. Bourguet, M. Machmoum, P. Guerin, and P. Rhein, "Reliability analysis of the collection system of an offshore wind farm," in 2014 Ninth International Conference on Ecological Vehicles and Renewable Energies (EVER), 2014, pp. 1-6.

[13] L. He, M. Zhou, F. Guo, and H. Yi, "Comparative study on reliability of wind farm collector systems," in International Conference on Renewable Power Generation (RPG 2015), 2015, pp. 1-5.

[14] O. Dahmani, S. Bourguet, M. Machmoum, P. Guerin, P. Rhein, and L. Josse, "Optimization and reliability evaluation of an offshore wind farm architecture," IEEE Transactions on Sustainable Energy, vol. 8, no. 2, pp. 542-550, 2017.

[15] S. Sulaeman, M. Benidris, J. Mitra, and C. Singh, "A wind farm reliability model considering both wind variability and turbine forced outages," IEEE Transactions on Sustainable Energy, vol. 8, no. 2, pp. 629-637, 2017.

[16] F. Castro Sayas and R. N. Allan, "Generation availability assessment of wind farms," IEE Proceedings - Generation, Transmission and Distribution, vol. 143, no. 5, pp. 507-518, 1996.

[17] R. Billinton and Y. Gao, "Multistate wind energy conversion system models for adequacy assessment of generating systems incorporating wind energy," IEEE Transactions on Energy Conversion, vol. 23, no. 1, pp. 163-170, 2008.

[18] H. Kim, C. Singh, and A. Sprintson, "Simulation and estimation of reliability in a wind farm considering the wake effect," IEEE Transactions on Sustainable Energy, vol. 3, no. 2, pp. 274-282, 2012.

[19] A. S. Dobakhshari and M. Fotuhi-Firuzabad, "A reliability model of large wind farms for power system adequacy studies," IEEE Transactions on Energy Conversion, vol. 24, no. 3, pp. 792-801, 2009.

[20] B. Wang et al., "An Analytical Approach to Evaluate the Reliability of Offshore Wind Power Plants Considering Environmental Impact," IEEE Transactions on Sustainable Energy, vol. 9, no. 1, pp. 249-260, 2018.

[21] B. Wang, X. Wang, X. Wang, and C. Shao, "Reliability evaluation of offshore wind power plants considering environmental effect," in 2017 IEEE Power Energy Society General Meeting, 2017, pp. 1-5.

[22] R. Billinton, B. Karki, R. Karki, and G. Ramakrishna, "Unit commitment risk analysis of wind integrated power systems," IEEE Transactions on Power Systems, vol. 24, no. 2, pp. 930-939, 2009.

[23] Y. Ding, C. Singh, L. Goel, J. Østergaard, and P. Wang, "Short-term and medium-term reliability evaluation for power systems with high penetration of wind power," IEEE Transactions on Sustainable Energy, vol. 5, no. 3, pp. 896-906, 2014.

[24] R. Billinton and W. Wangdee, "Reliability-based transmission reinforcement planning associated with large-scale wind farms," IEEE Transactions on Power Systems, vol. 22, no. 1, pp. 34-41, 2007.

[25] R. Billinton and R. N. Allan, Reliability Evaluation of Power Systems, 2nd ed. New York, NY: Plenum Press, 1994.

[26] J. Pérez-Rúa, M. Stolpe, K. Das, and N. A. Cutululis, "Global optimiza- 
tion of offshore wind farm collection systems," IEEE Transactions on Power Systems, vol. 35, no. 3, pp. 2256-2267, 2020.

[27] R. A. Howard, Dynamic Probabilistic Systems: Volume I, 2nd ed. Mineola, NY: Dover Publications, Inc., 1971.

[28] Y. Guo, H. Gao, and Q. Wu, "A combined reliability model of vsc-hvdc connected offshore wind farms considering wind speed correlation," IEEE Transactions on Sustainable Energy, vol. 8, no. 4, pp. 1637-1646, 2017.

[29] B. Kerres, K. Fischer, and R. Madlener, "Economic evaluation of maintenance strategies for wind turbines: a stochastic analysis," IET Renewable Power Generation, vol. 9, no. 7, pp. 766-774, 2015.

[30] K. Fischer, F. Besnard, and L. Bertling, "Reliability-centered maintenance for wind turbines based on statistical analysis and practical experience," IEEE Transactions on Energy Conversion, vol. 27, no. 1, pp. 184-195, 2012.

[31] J. Carroll, A. McDonald, and D. McMillan, "Reliability comparison of wind turbines with DFIG and PMG drive trains," IEEE Transactions on Energy Conversion, vol. 30, no. 2, pp. 663-670, 2015.

[32] J. Carroll, A. McDonald, I. Dinwoodie, D. McMillan, M. Revie, and I. Lazakis, "Availability, operation and maintenance costs of offshore wind turbines with different drive train configurations," Wind Energy, vol. 20, pp. 361-378, 2017.

[33] J. J. B. Gill, "Forecasting underground electric cable faults using the Crow AMSAA model," Asset Management \& Maintenance Journal, vol. 24, p. 40, 2011.

[34] O. Yevkin and V. Krivtsov, "Comparative analysis of optimal maintenance policies under general repair with underlying weibull distributions," IEEE Transactions on Reliability, vol. 62, no. 1, pp. 82-91, 2013.

[35] M. Buhari, V. Levi, and S. K. E. Awadallah, "Modelling of ageing distribution cable for replacement planning," IEEE Transactions on Power Systems, vol. 31, no. 5, pp. 3996-4004, 2016.

[36] Z. Tang, W. Zhou, J. Zhao, D. Wang, L. Zhang, H. Liu, Y. Yang, and C. Zhou, "Comparison of the weibull and the Crow-AMSAA Model in prediction of early cable joint failures," IEEE Transactions on Power Delivery, vol. 30, no. 6, pp. 2410-2418, 2015.

[37] R. Billinton and P. Alukal, "Availability analysis in a cable transmission system with repair restrictions," IEEE Transactions on Power Systems, vol. 3, no. 3, pp. 872-878, 1988.

[38] B. W. Tuinema, "Reliability of transmission networks: Impact of EHV underground cables \& interaction of offshore-onshore networks," Ph.D. dissertation, TU Delft, 2017.

[39] L. Cheng, H. Feng, Y. Chang, and C. Singh, "Reliability analysis of HTS cable systems," IEEE Transactions on Power Delivery, vol. 30, no. 3, pp. 1251-1259, 2015.

[40] A. Lisnianski, I. Frenkel, and Y. Ding, Multi-State System Reliability Analysis and Optimization for Engineers and Industrial Managers. London: Springer, 2010.

[41] M. Zhao, Z. Chen, and F. Blaabjerg, "Generation ratio availability assessment of electrical systems for offshore wind farms," IEEE Transactions on Energy Conversion, vol. 22, no. 3, pp. 755-763, 2007.

[42] "Anholt Offshore Wind Farm," Accessed: Aug. 03, 2020. [Online]. Available: https://en.wikipedia.org/wiki/Anholt_Offshore_Wind_Farm

[43] J. Bezanson, A. Edelman, S. Karpinski, and V. B. Shah, "Julia: A fresh approach to numerical computing," SIAM Review, vol. 59, no. 1, pp. 65-98, 2017.

[44] "Offshore wind data," Library Catalog: Ørsted.com. [Online]. Available: https://orsted.com/en/our-business/offshore-wind/wind-data

[45] O. Siirto, J. Vepsäläinen, A. Hämäläinen, and M. Loukkalahti, "Improving reliability by focusing on the quality and condition of mediumvoltage cables and cable accessories," CIRED - Open Access Proceedings Journal, vol. 2017, no. 1, pp. 229-232, 2017.

[46] S. Lumbreras, A. Ramos, and P. Sánchez-Martin, "Offshore wind farm electrical design using a hybrid of ordinal optimization and mixedinteger programming," Wind Energy, vol. 18, no. 12, pp. 2241-2258, 2015.

[47] "National Report: Denmark Status for 2017," Danish Utility Regulator, Denmark, Tech. Rep., 2018, Accessed: Sep. 12, 2020. [Online]. Available: https://www.ceer.eu/documents/104400/6319351/ C18_NR_Denmark-EN/9b5189c6-ffb4-8faa-e60b-ebc05a81812c
[48] J. Campos, T. Serebrisky, and A. Suárez-Alemán, "Time Goes By: Recent Developments on the Theory and Practice of the Discount Rate," Inter-American Development Bank, Tech. Rep., Sep. 2015.

Gayan Abeynayake (Graduate Student Member, IEEE) received the B.Sc.Eng. degree (Hon.) in electrical and electronic engineering and the M.Sc.Eng. degree in power, energy systems and high voltage from the University of Peradeniya, Sri Lanka, in 2011 and 2016, respectively. Since April 2018, he has been working toward the Ph.D. degree in electrical engineering with Cardiff University, Cardiff, U.K as a Marie Curie Early Stage Research Fellow attached to InnoDC Project. His main research interests include power systems, and power electronics reliability, grid integration of renewable energy, and power system planning. He was the recipient of the Prof. E.O.E Pereira Gold Medal for the Most Outstanding Engineering Graduate at the General Convocation 2012 and received the 1st prize of the IEEE PES UKRI presentation competition, Cardiff, in 2018. He is the Secretary of the IEEE PELS Society U.K.\&I chapter for the term 2020-2021.

Tom Van Acker received the M.Eng., M.Sc. and Ph.D. degrees in Electrical Engineering from KU Leuven, Leuven, Belgium in 2012, 2014 and 2020, respectively. Currently, he is a power system expert at BASF Antwerp. His main areas of research interest are stochastic processes and optimization in a power system context.

Dirk Van Hertem (Senior Member, IEEE) graduated as a M.Eng. in 2001 from the KHK, Geel, Belgium and as a M.Sc. in Electrical Engineering from the KU Leuven, Belgium in 2003. In 2009, he has obtained his $\mathrm{PhD}$, also from the KU Leuven. In 2010, Dirk Van Hertem was a member of EPS group at the Royal Institute of Technology (KTH), in Stockholm. Since spring 2011 he is back at the University of Leuven where he is an associate professor in the ELECTA group. His special fields of interest are decision support for grid operators, power system operation and control in systems with FACTS and HVDC and building the transmission system of the future, including offshore grids and the supergrid concept. The research activities of Prof. Van Hertem are all part of the EnergyVille research center, where he leads the Electrical Networks activities. Dr. Van Hertem is an active member of both IEEE (PES and IAS) and Cigré. He currently chair of the steering committee of the ISGT Europe conference. He was the general chair of the IEEE EnergyCon 2016 conference.

Jun Liang (Senior Member, IEEE) received the B.Sc. degree in electric power system and its automation from the Huazhong University of Science and Technology, Wuhan, China, in 1992, and the M.Sc. and Ph.D. degrees in electric power system and its automation from China Electric Power Research Institute (CEPRI), Beijing, China, in 1995 and 1998, respectively. From 1998 to 2001, he was a Senior Engineer with CEPRI, from 2001 to 2005, he was a Research Associate with Imperial College London, London, U.K., and from 2005 to 2007, he was a Senior Lecturer with the University of Glamorgan, Wales, U.K. He is a Professor in power electronics with the School of Engineering, Cardiff University, Cardiff, U.K. He is the Coordinator and Scientist-in-Charge of two European Commission Marie-Curie Action ITN/ETN projects, MEDOW $(€ 3.9 \mathrm{M})$ and InnoDC $(€ 3.9 \mathrm{M})$. His research interests include HVDC, MVDC, FACTS, power system stability control, power electronics, and renewable power generation. Prof. Liang is a Fellow of the Institution of Engineering and Technology, the Chair of the IEEE U.K. and Ireland Power Electronics Chapter, an Editorial Board Member of the CSEE JPES, and the Editor of the IEEE TRANSACTIONS ON SUSTAINABLE ENERGY. 